\title{
Complex Potential Functions and Integro-Differential Equation in Elastic Media Problem in Presence of Heat
}

\author{
M. A. Seddeek ${ }^{1}$, M. A. Abdou, ${ }^{2, *}$, W. G. El-Sayed ${ }^{2}$, F. M. El-Saedy ${ }^{3}$ \\ ${ }^{1}$ Department of Mathematics, Deanship of Educational Services, Al-Qassim Univ ersity, Saudi Arabia \\ ${ }^{2}$ Department of Mathematics, Faculty of Science, Alexandria University, Egy pt \\ ${ }^{3}$ Department of Mathematics, Faculty of Science, Umm Al-Qura University, Makkah, Saudi Arabia
}

\begin{abstract}
This paper covered the study of the boundary value problem for isotropic homogeneous perforated infinite elastic media in presence of uniform flow of heat. For this, we considered the problem of a thin infinite plate of specific thickness with a curvilinear hole where the origin lie inside the hole is conformally mapped outside a unit circle by means of a specific rational mapping. The complex variable method has been applied and it transforms the problem to the integro-differential equation with Cauchy kernel that can be solved to find two complex potential functions which called Gaursat functions. Moreover, the three stress components $\sigma_{x x}, \sigma_{y y}, \sigma_{x y}$ for the boundary value problem in the thermoelasticity plane are obtained. Many special cases of the conformal mapping and three applications for different cases are discussed and many main results derived from the work.
\end{abstract}

Keywords Boundary Value Problem of Infinite Plate Weakened by a Hole, Conformal Mapping, Integro-Differential Equation with Discontinuous Kernel, Complex Potential Functions

\section{Introduction}

Several authors wrote about the boundary value problems and their applications in many different sciences, see Gakhov [1], Ciarlet et al.[2], Zebib[3] and Saito and Yamamto[4]. From these problems, we established contact problems and mixed problems in the theory of elasticity, see Colton and Kress[5], and Abdou[6]. In Abdou[6,7], Abdou and Khamis [8] and Abdou and Khar-Eldin [9],Comp lex variable method is used to express the solutions of these problems in the form of power series by applied Laurant's theorem. On the other hand, some of authors applied the complex variable method to obtain the solutions in the form two complex potential functions which called Gaursat functions by using the properties of Cauchy integrals on the circle, or on any region mapped to outside a unit circle $\gamma$ by means of a general rational mapping $z=c \omega(\zeta)$ where, $\omega^{\prime}(\zeta)$ doesn't vanish or become infinite outside the unit circle.

In thermoelastic problems for elastic media, the first and second boundary value problems are equivalent to finding two analytic functions $\phi(z)$ and $\psi(z)$ of one complex argument $z=x+i y$. These functions must satisfy the boundary conditions,

$$
K \phi_{1}(t)-t \overline{\phi_{1}^{\prime}(t)}-\overline{\psi_{1}(t)}=f(t)
$$

* Corresponding author:

abdella_777@yahoo.com (M. A. Abdou)

Published online at http://journal.sapub.org/ajfd

Copyright (C) 2012 Scientific \& Academic Publishing. All Rights Reserved where $t$ denotes the affix of a point on the boundary. In terms of $z=c \omega(\zeta), c>0, \omega^{\prime}(\zeta)$ does not vanish or become infinite for $|\zeta|>1$, the infinite region mapped to outside a unit circle $\gamma$.

For the first fundamental boundary value problem or it called the stress boundary value problem we have $\mathrm{K}=-1$ and $f(t)$ is a given function of stress. While for $K=k>1$ and $f(t)$ is a given function of displacement which called the thermal conductivity then eq.(1.1) called the second fundamental boundary value problems or the displacement boundary value problems. The books written by Noda et al.[10], Hetnarski and Ignaczak[11], Parkus[12] and Popov[13] contain many different methods to solve the problems in the theory of elasticity in one, two and three dimensions.

The complex potential functions $\phi_{1}(t)$ and $\psi_{1}(t)$ take the following form, see Parkus [12]

$$
\phi_{1}(\zeta)=-\frac{S_{x}+i S_{y}}{2 \pi(1+k)} \ln \zeta+c \Gamma \zeta+\phi(\zeta)
$$

and,

$$
\psi_{1}(\zeta)=k \frac{\left(S_{x}-i S_{y}\right)}{2 \pi(1+k)} \ln \zeta+c \Gamma^{*} \zeta+\psi(\zeta)
$$

where, $S_{x}, S_{y}$ are the components of the resultant vector of all external forces acting on the boundary and $\Gamma, \Gamma^{*}$ are complex constants. Generally the two complex functions $\varphi(\zeta)$ and $\psi(\zeta)$ are single value analytic functions within the region outside the unit circle $\gamma$ and $\varphi(\infty)=0, \psi(\infty)=0$. 
In [14], Muskhelishvili used the rational mapping, $z=c\left(\zeta+m \zeta^{-1}\right), \quad c>0, m$ is real number,

to solving the problem of infinite plate weakened by an elliptic hole.

El-Sirafy and Abdou in [15], used the rational mapping,

$$
z=c \frac{\zeta+m \zeta^{-1}}{1-n \zeta^{-1}}, c>0,|n|<1
$$

to solve the first and second fundamental problems for the infinite plate with general curvilinear hole $C$ confrmally mapped on the domain outside a unit circle.

The rational mapping,

$$
z=c\left(\zeta+m_{1} \zeta^{-1}+m_{2} \zeta^{-2}\right) \quad, \quad 0 \leq m_{j} \leq 1, j=1,2
$$

is used by Abdou and Khamis [8] to obtain the solution of the problem of an infinite plate with a curvilinear hole having three poles and they take the circle shape, ellipse shape and square shape as a special cases of the holes.

Also, the conformal mapping function,

$$
z=c \frac{\zeta+m \zeta^{-1}}{\left(1-n_{1} \zeta^{-1}\right)\left(1-n_{2} \zeta^{-1}\right)} \quad, \quad c>0,
$$

is studied completely by England [16].

In this paper, we consider the boundary value problem for isotropic homogeneous perforated infinite elastic media in presence of uniform flow of heat. Then, we use the more general shape of conformal mapping to obtain the complex potential functions for the problem in the form integro- differential equation with singular kennel. Many special cases are obtained and several applications are discussed fro $\mathrm{m}$ the work.

This study is useful for researchers who work on the studies of petroleum tubes industry or water or gas. It also benefits the physics scientists who work on the study of the ozone hole.

\section{Formulation of the Problem}

Consider a thin infinite plate of thickness $h$ with a curvilinear hole $C$, where the origin lie inside the hole is conformally mapped on the domain outside a unit circle $\gamma$ by means of a rational mapping,

$$
z=c \frac{\zeta+m_{1} \zeta^{-1}+m_{2} \zeta^{-2}}{\left(1-n_{1} \zeta^{-1}\right)\left(1-n_{2} \zeta^{-1}\right)} \quad, \quad c>0, n_{1} \neq n_{2}
$$

where $z^{\prime}(\zeta)$ does not vanish or become infinite outside the unit circle $\gamma$. If a temperature distribution $\Theta=q y$ is following uniformly in the direction of the negative $y$-axis, where the increasing a temperature distribution $\Theta$ is assumed to be constant a cross the thickness of the plate, i.e. $\Theta=\Theta(x, y)$, and $q$ is the constant temperature gradient. The uniform flow of heat is distributed by the presence of an insulated curvilinear hole $C$.

The heat equation satisfies the relation,

$$
\nabla^{2} \Theta=0 \quad, \quad \nabla^{2}=\frac{\partial^{2}}{\partial x^{2}}+\frac{\partial^{2}}{\partial y^{2}}
$$

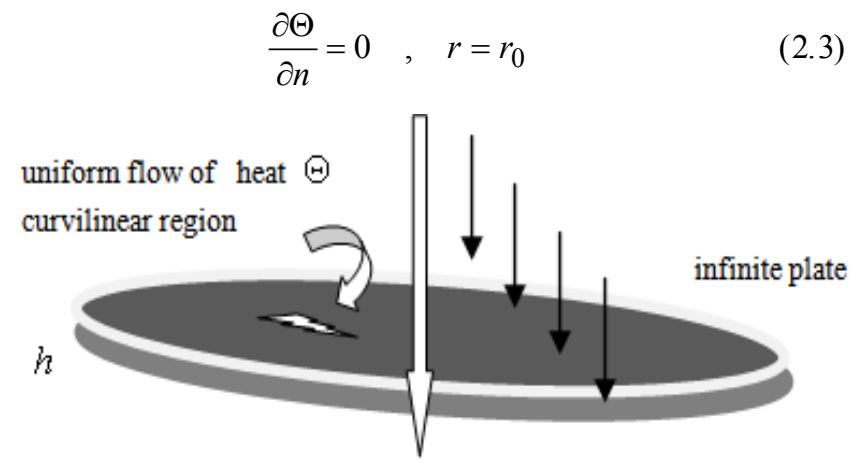

Figure (1).

where $n$ is the unit vector perpendicular to the surface.

Neglecting the variation of the strain and the stress with respect to the thickness of the plate, the thermoelastic potential $\Phi$ satisfies the formula, see Parkus [12]

$$
\nabla^{2} \Phi=(1+v) \alpha \Theta
$$

where $\alpha$ is a scalar which present the coefficient of the thermal expansion and $v$ is Poisson's ratio. Assume the force of the plate is free of applied loads.

In this case, the formula (1.1) for the first and second boundary value problems respectively take the following form,

$$
\begin{gathered}
\varphi_{1}(t)+t \overline{\varphi_{1}^{\prime}(t)}+\overline{\psi_{1}(t)}=\frac{\partial \Phi}{\partial x}+i \frac{\partial \Phi}{\partial y}+\frac{1}{2 G} \int_{0}^{s}[i X(s)-Y(s)] d s+c(2.5) \\
k \phi_{1}(t)-t \overline{\phi_{1}^{\prime}(t)}-\overline{\psi_{1}(t)}=u+i v-\frac{\partial \Phi}{\partial x}-i \frac{\partial \Phi}{\partial y}
\end{gathered}
$$

where the applied stresses $X(s)$ and $Y(s)$ are prescribed on the boundary of the plane $s$ is the length measured from an arbitrary point, $u$ and $v$ are the displacement components, $G$ is the shear modulus. Also, here the applied stresses $X(s)$ and $Y(s)$ must satisfy the following, see Parkus [12]

$$
\begin{aligned}
& X(s)=\sigma_{x x} \frac{d y}{d s}-\sigma_{x y} \frac{d x}{d s} \\
& Y(s)=\sigma_{y x} \frac{d y}{d s}-\sigma_{y y} \frac{d x}{d s}
\end{aligned}
$$

where $\sigma_{x x}, \sigma_{x y}$ and $\sigma_{y y}$ are the components of stresses which are given by the following relations,

$$
\begin{gathered}
\sigma_{x x}-\sigma_{y y}+2 i \sigma_{x y}= \\
2 G\left[\frac{\partial^{2} \Phi}{\partial y^{2}}-\frac{\partial^{2} \Phi}{\partial x^{2}}+2 i \frac{\partial^{2} \Phi}{\partial x \partial y}\right]+4 G\left[\bar{z} \varphi^{\prime \prime}(z)+\psi^{\prime \prime}(z)\right] \\
\sigma_{x x}+\sigma_{y y}=4 G\left[\operatorname{Re} \phi^{\prime}(z)-\lambda \Theta\right]
\end{gathered}
$$

where $\lambda=\frac{\alpha}{2}(1+v)$ is the coeffic ient of heat transfer.

The rational mapping $z=c \omega(\zeta)$ maps the boundary $C$ of the given region occupied by the middle plane of the plate in the $z$-plane onto the unit circle $\gamma$ in the $\zeta$-plane. Curvilinear coordinates $(\rho, \theta)$ are thus introduce into the $z$ - plane which are the maps of the polar conditions in the $\zeta$ - plane as given by $\zeta=\rho e^{i \theta}, 0 \leq \theta \leq 2 \pi$. By using the transformation $z=c \omega(\zeta)$, Eq.(1.1) reduce to, 
$K \varphi_{1}(c \omega(\zeta))-\frac{\omega(\zeta)}{\overline{\omega^{\prime}(\zeta)}} \overline{\varphi_{1}(c \omega(\zeta))}-\overline{\psi_{1}(c \omega(\zeta))}=f(c \omega(\zeta))(2.11)$

The last formula represent the first and second boundary value problems in $\zeta$ - plane.

\section{The Rational Mapping}

The rational mapping (2.1) maps the curvilinear hole $C$ in $z$ - plane onto the domain of outside unit circle in $\zeta-$ plane under the condition $\omega^{\prime}(\zeta)$ does not vanish or become infinite outside the unit circle $\gamma$. The following graphs give the different shapes of the rational mapping (2.1).

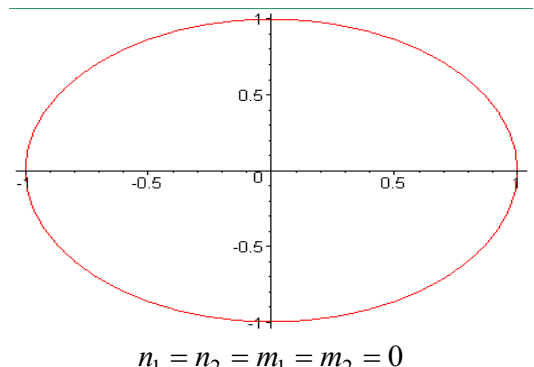

Figure (2)

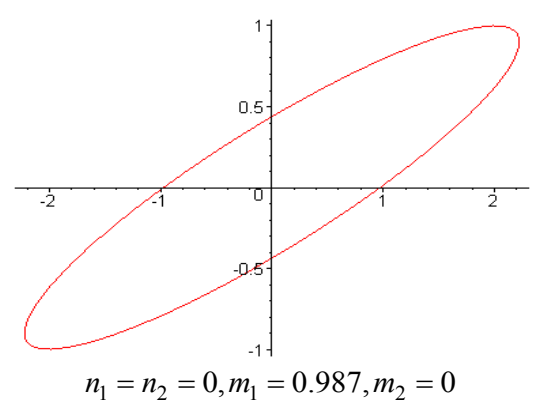

Figure (3)

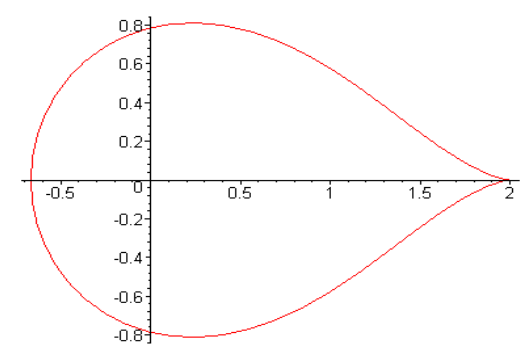

$n_{1}=0.5, n_{2}=m_{1}=m_{2}=0$

Figure (4)

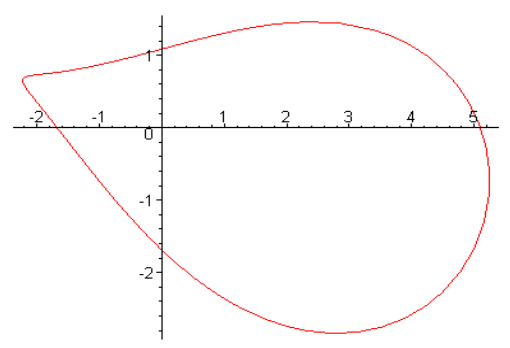

$n_{1}=0.4, n_{2}=0,=m_{1}=2-i, m_{2}=0$
Figure (5)

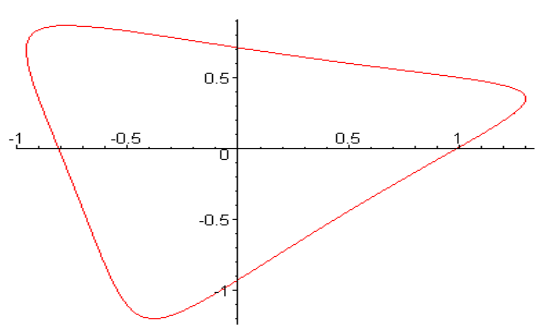

$n_{1}=n_{2}=0$,

$m_{1}=0.05+0.05 i, m_{2}=0.2+0.2 i$

Figure (6)

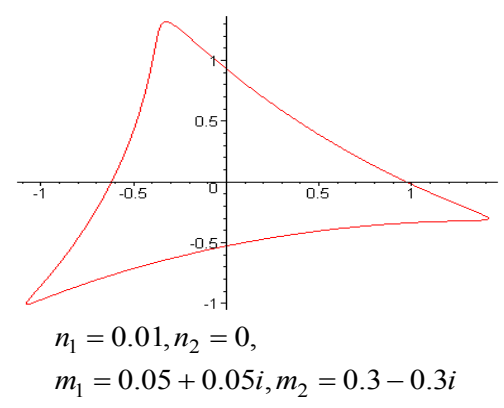

Figure (7)

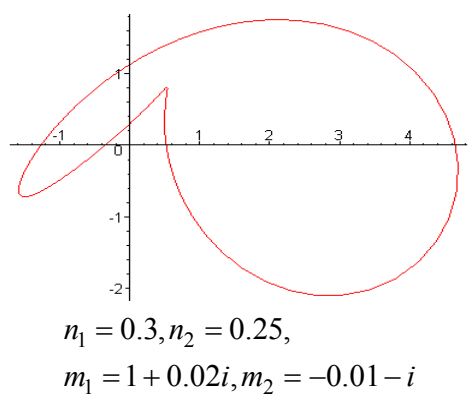

Figure (8)

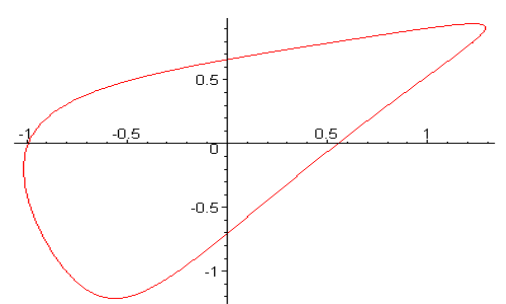

$n_{1}=0.002, n_{2}=0.001$,

$m_{1}=0.04+0.4 i, m_{2}=0.02+0.2 i$

Figure (9)

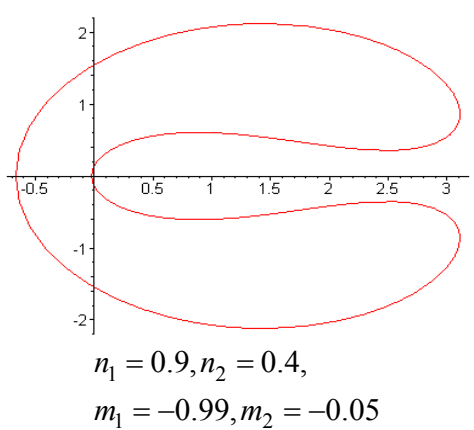


Figure (10)

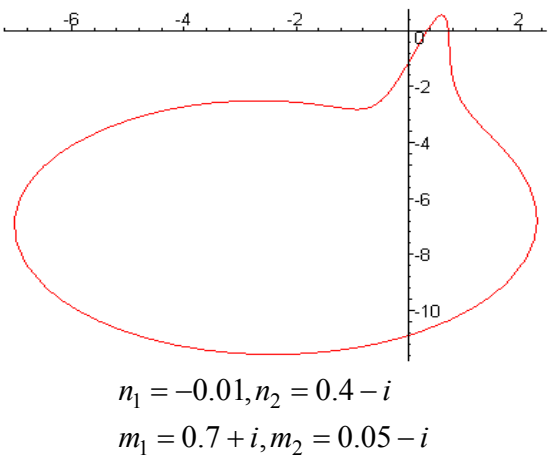

Figure (11)

\section{Method of Solution}

In this section, we use the complex variable method to obtain the two complex functions, Goursat functions, $\varphi(\zeta)$ and $\psi(\zeta)$.Moreover, the three stress components $\sigma_{x y}, \sigma_{x x}$ and $\sigma_{y y}$ will be complete determined.

\subsection{The Stress Components}

The solution of Eq. (2.2) is given by,

$$
\Theta=q\left[R+\frac{r_{0}^{2}}{R}\right], \quad R=\sqrt{x^{2}+y^{2}}
$$

By substitute Eq.(4.1) in Eq.(2.4) and using the definition of $\nabla^{2} \Phi$ in polar coordinates the thermoelastic potential function take the form,

$$
\Phi=\frac{(1+v) \alpha q r_{0}^{2}}{4} \ln z R^{2}
$$

Also, the stresses components can be adapted in the form,

$$
\begin{gathered}
\sigma_{x x}=2 G\left[-\frac{1}{2}\left(\frac{\partial^{2} \Phi}{\partial y^{2}}-\frac{\partial^{2} \Phi}{\partial x^{2}}+2 \lambda \Theta\right)\right. \\
\left.+\operatorname{Re}\left[2 \varphi^{\prime}(z)-\bar{z} \varphi^{\prime \prime}(z)-\psi^{\prime \prime}(z)\right]\right] \\
\sigma_{y y}=2 G\left[\frac{1}{2}\left(\frac{\partial^{2} \Phi}{\partial y^{2}}-\frac{\partial^{2} \Phi}{\partial x^{2}}-2 \lambda \Theta\right)+\operatorname{Re}\left[2 \varphi^{\prime}(z)+\bar{z} \varphi^{\prime \prime}(z)+\psi^{\prime \prime}(z)\right]\right],
\end{gathered}
$$

and,

$$
\sigma_{x y}=2 G\left[\frac{\partial^{2} \Phi}{\partial x \partial y}+\operatorname{Im}\left(\bar{z} \phi^{\prime \prime}(z)+\psi^{\prime \prime}(z)\right)\right] .
$$

Eqs.(4.3), (4.4) and (4.5) after some derivatives and algebraic relations adapted as,

$$
\begin{gathered}
\sigma_{x x}=2 G\left[-\eta\left(z^{2}+4 z \bar{z}+\bar{z}^{2}\right) \operatorname{Im} z+\operatorname{Re}\left[2 \varphi^{\prime}(z)-M(z, \bar{z})\right]\right], \\
\sigma_{y y}=2 G\left[\eta\left(z^{2}+4 z \bar{z}+\bar{z}^{2}\right) \operatorname{Im} z+\operatorname{Re}\left[2 \varphi^{\prime}(z)+M(z, \bar{z})\right],\right.
\end{gathered}
$$

and,

$$
\sigma_{x y}=2 G\left[\eta\left(z \bar{z}-2(\operatorname{Im} z)^{2} \operatorname{Re} z+\operatorname{Im} M(z, \bar{z})\right],\right.
$$

where,

$$
\eta=\frac{(1+v) r_{0}^{2}}{2(z \bar{z})^{2}}
$$

and,

$$
M(z, \bar{z})=\bar{z} \phi^{\prime \prime}(z)+\psi^{\prime}(z) .
$$

After determine the Goursat functions the components of stress are completely determined.

\subsection{Gours at Functions}

To obtain the two complex potential functions, Goursat functions by using the conformal mapping (2.1) in the boundary condition(1.1), we write the expression $\frac{\omega(\zeta)}{\omega^{\prime}\left(\zeta^{-1}\right)}$ in the form,

$$
\frac{\omega(\zeta)}{\omega^{\prime}\left(\zeta^{-1}\right)}=\alpha(\zeta)+\beta\left(\zeta^{-1}\right)
$$

where,

$$
\alpha(\zeta)=\frac{h_{1}}{\zeta-n_{1}}+\frac{h_{2}}{\zeta-n_{2}},
$$

and,

$$
\begin{aligned}
& h_{j}=\frac{\left(n_{j}^{3}+m_{1} n_{j}+m_{2}\right)\left(1-n_{j}^{2}\right)^{2}\left(1-n_{1} n_{2}\right)^{2}}{\left(n_{j}-n_{j \pm 1}\right)\left[1-2\left(n_{1}+n_{2}\right) n_{j}-\left(m_{1}-3 n_{1} n_{2}\right) n_{j}^{2}\right.}, j=0,1 \\
& \left.\quad-2 m_{2} n_{j}^{3}+\left(n_{1} n_{2} m_{1}+n_{1} m_{2}+n_{2} m_{2}\right) n_{j}^{4}\right] \\
& \beta\left(\zeta^{-1}\right) \text { is a regular function for }|\zeta|>1 . \\
& \frac{\omega(\zeta)}{\omega^{\prime}\left(\zeta^{-1}\right)} \text { has a singularity at } \zeta=n_{1} \text { and } \zeta=n_{2} .
\end{aligned}
$$

Using Eq.(1.2) and Eq.(1.3) in Eq.(2.11), we get

$$
\begin{aligned}
& K c \Gamma \zeta+K \varphi(\zeta)+\frac{\omega(\zeta)}{\omega^{\prime}\left(\zeta^{-1}\right)}\left[\frac{\left.S_{x}-i S_{y}\right)}{2 \pi(1+k)} \zeta\right. \\
& \left.-c \bar{\Gamma}-\overline{\varphi^{\prime}(\zeta)}\right]+c \overline{\Gamma^{*}} \zeta^{-1}-\overline{\psi(\zeta)}=F(\zeta)
\end{aligned}
$$

Using Eq.(4.12) in Eq.(4.14), we have

$$
\begin{aligned}
& K \varphi(\zeta)-\alpha(\zeta) \overline{\varphi^{\prime}(\zeta)}-\overline{\psi(\zeta)}-\overline{\beta(\zeta) \varphi^{\prime}(\zeta)} \\
& -N(\zeta)\left[\alpha(\zeta)+\overline{\beta(\zeta)]}=F(\zeta)-c K \Gamma \zeta-c \overline{\Gamma^{*}} \zeta^{-1}\right.
\end{aligned}
$$

Taking $\zeta=\sigma$, we get

$$
K \phi(\sigma)-\alpha(\sigma) \overline{\phi^{\prime}(\sigma)}-\overline{\psi_{*}(\sigma)}=F_{*}(\sigma),
$$

where,

$$
\psi_{*}(\zeta)=\psi(\zeta)+\beta(\zeta) \phi^{\prime}(\zeta),
$$

$$
\begin{gathered}
F_{*}(\zeta)=F(\zeta)-c K \Gamma \zeta-c \overline{\Gamma^{*}} \zeta^{-1}+N(\zeta)[\alpha(\zeta)+\overline{\beta(\zeta)}] \\
N(\zeta)=c \bar{\Gamma}-\frac{S_{x}-i S_{y}}{2 \pi(1+k)} \zeta
\end{gathered}
$$

and,

$$
F(\zeta)=f(c \omega(\zeta))=f(t) .
$$

Assume the function $F(\sigma)$ with its derivatives must satisfy Hölder condition. Our aim is to determine the functions $\phi(\zeta)$ and $\psi(\zeta)$ for the various boundary value problems. For this multiply both side of Eq.(4.16) by $\frac{d \sigma}{2 \pi i(\sigma-\zeta)}$ where $\zeta$ is any point the interior of $\gamma$ and integral over the circle, we obtain 
$\frac{K}{2 \pi i} \int_{\gamma} \frac{\varphi(\sigma)}{(\sigma-\zeta)} d \sigma-\frac{1}{2 \pi i} \int_{\gamma} \frac{\alpha(\sigma) \overline{\varphi^{\prime}(\sigma)}}{(\sigma-\zeta)} d \sigma-\frac{1}{2 \pi i} \int_{\gamma} \frac{\overline{\psi_{*}(\sigma)}}{(\sigma-\zeta)} d \sigma$

$=\frac{1}{2 \pi i} \int_{\gamma} \frac{F(\sigma)}{(\sigma-\zeta)} d \sigma$

Using Eqs.(4.17),(4.18) and (4.19) in Eq.(4.21) then applying the properties of Cauchy integral to have

$$
\begin{aligned}
& -K \varphi(\zeta)-\frac{1}{2 \pi i} \int_{\gamma} \frac{\alpha(\sigma) \overline{\varphi^{\prime}(\sigma)}}{(\sigma-\zeta)} d \sigma \\
& -\frac{1}{2 \pi i} \int_{\gamma} \frac{N(\sigma) \alpha(\sigma)}{(\sigma-\zeta)} d \sigma=A(\zeta)-c \overline{\Gamma^{*}} \zeta^{-1},
\end{aligned}
$$

where,

$$
A(\zeta)=\frac{1}{2 \pi i} \int_{\gamma} \frac{F(\sigma)}{(\sigma-\zeta)} d \sigma .
$$

The formula (4.22) represents the integro-differential equation of second kind with Cauchy kernel. The references, Fedotov[17], Hanyga and Seredyńska[18], Bavula[19] and AL-Jawary and Wrobel[20], contain many different methods to solve this kind of the equations analytically and numerically in one, two and three dimensions.

To obtain the integral terms of Eq. (4.22) we use Eq. (4.12) and then apply the residue theorem to have,

$$
\frac{1}{2 \pi i} \int_{\gamma} \frac{\alpha(\sigma) \overline{\phi^{\prime}(\sigma)}}{(\sigma-\zeta)} d \sigma=c \sum_{j=1}^{2} \frac{h_{j} b_{j}}{n_{j}-\zeta},
$$

where $b_{j}, j=1,2$ are complex constants.

Also,

$$
\frac{1}{2 \pi i} \int_{\gamma} \frac{N(\sigma) \alpha(\sigma)}{(\sigma-\zeta)} d \sigma=\sum_{j=1}^{2} \frac{N\left(n_{j}\right) h_{j}}{n_{j}-\zeta} .
$$

So, Eq.(4.22) reduce to,

$$
-K \varphi(\zeta)=A(\zeta)-c \overline{\Gamma^{*}} \zeta^{-1}+\sum_{j=1}^{2} \frac{h_{j}}{n_{j}-\zeta}\left[N\left(n_{j}\right)+c b_{j}\right] .
$$

To determine the constants $b_{j}$, differentiating Eq.(4.26) with respect to $\zeta$ and substituting in Eq.(4.24), to get

$$
\begin{aligned}
& \frac{1}{2 \pi i} \int_{\gamma} \frac{\alpha(\sigma)}{(\sigma-\zeta)}\left[-\overline{A^{\prime}(\sigma)}-c \Gamma^{*} \sigma^{2}\right. \\
& \left.-\sum_{j=1}^{2} \frac{h_{j}}{\left(n_{j} \sigma-1\right)^{2}}\left[\overline{N\left(n_{j}\right)}+c \bar{b}_{j}\right]\right] d \sigma=c K \sum_{j=1}^{2} \frac{h_{j} b_{j}}{n_{j}-\zeta}
\end{aligned}
$$

Substituting Eq.(4.12) in Eq.(4.27), then using the properties of Cauchy integral and applying the residue theorem at the singular points, we obtain

$$
c K b_{j}+c \Gamma^{*} n_{j}^{2}+h_{j} d_{j}\left(\overline{N\left(n_{j}\right)}+c \bar{b}_{j}\right)=-\overline{A\left(n_{j}\right)},
$$

where,

$$
d_{j}=\frac{n_{j}^{2}}{\left(1-n_{j}^{2}\right)^{2}} \quad, \quad j=1,2 .
$$

The last equation can be written in the form,

$$
c K b_{j}+c h_{j} d_{j} \bar{b}_{j}=E_{j},
$$

where,

$$
E_{j}=-\overline{A^{\prime}\left(n_{j}\right)}-c \Gamma^{*} n_{j}^{2}-h_{j} d_{j} \overline{N\left(n_{j}\right)} .
$$

Taking the complex conjugate of Eq.(4.30) we get,

$$
c K \bar{b}_{j}+c h_{j} d_{j} b_{j}=\bar{E}_{j} \text {. }
$$

From Eq.(4.30) and Eq.(4.32) we have,

$$
b_{j}=\frac{K E_{j}-h_{j} d_{j} \bar{E}_{j}}{c\left(K^{2}-h_{j}^{2} d_{j}^{2}\right)} \quad, \quad j=1,2 .
$$

To obtain the complex functions $\psi(\zeta)$ we have form Eq.(4.17) after substituting the expression of $\psi_{*}(\sigma)$ and $F_{*}(\sigma)$, and taking the complex conjugate of the resulting equation after using the expression of $\overline{\beta(\sigma)}$ to yields,

$$
\begin{aligned}
& \psi(\sigma)=-\overline{F(\sigma)}+c K \Gamma \sigma^{-1}-c \Gamma^{*} \sigma \\
& +K \overline{\varphi(\sigma)}-\overline{\alpha(\sigma)} \varphi_{*}(\sigma)-\frac{\overline{\omega(\sigma)}}{\omega^{\prime}(\sigma)} \varphi_{*}(\sigma) \\
& +\sum_{j=1}^{2} \frac{h_{j}}{1-n_{j} \sigma} \sigma \varphi_{*}(\sigma)
\end{aligned}
$$

where,

$$
\phi_{*}(\sigma)=\phi^{\prime}(\sigma)+\overline{N(\sigma)}
$$

and,

$$
\overline{N(\sigma)}=c \Gamma-\frac{S_{x}+i S_{y}}{2 \pi(1+k)} \sigma^{-1} .
$$

Multiplying both sides of Eq.(4.34) by $\frac{1}{2 \pi i(\sigma-\zeta)}$ where $\zeta$ is any point in the interior of $\gamma$ and integrating over the unit circle, then using the properties of Cauchy integral and calculate sum residue, we obtain

$$
\begin{aligned}
\psi(\zeta)= & c K \Gamma \zeta^{-1}-\frac{\omega\left(\zeta^{-1}\right)}{\omega^{\prime}(\zeta)} \varphi_{*}(\zeta) \\
& +\sum_{j=1}^{2} \frac{h_{j} \zeta}{1-n_{j} \zeta} \varphi_{*}\left(n_{j}^{-1}\right)+B(\zeta)-B
\end{aligned},
$$

where,

$$
B(\zeta)=\frac{1}{2 \pi i} \int_{\gamma} \frac{\overline{F(\sigma)}}{(\sigma-\zeta)} d \sigma,
$$

and,

$$
B=\frac{1}{2 \pi i} \int_{\gamma} \frac{\overline{F(\sigma)}}{\sigma} d \sigma .
$$

\section{Some Applications}

In this section, we assume different values of the given functions in the first or second fundamental boundary value problems. Then, we obtain the expression of Goursat functions. After that, the components of stresses can be calculated directly.

\subsection{Application 1: Curvilinear Hole for an Infinite Plate Subjected to Uniform Tensile Stress and Flowing Heat.}

For 
$K=-1, \Gamma=\frac{P}{4}, \Gamma^{*}=\frac{-P}{2} e^{-2 i \theta}, 0 \leq \theta \leq 2 \pi, S_{x}=S_{y}=f=0$,

we have,

$$
\left.\begin{array}{l}
A(\zeta)=0 \quad, \quad N(\zeta)=\frac{c P}{4}, \\
E_{j}=\frac{c P n_{j}^{2} e^{-2 i \theta}}{2}-\frac{c P}{4} h_{j} d_{j}, \\
\bar{E}_{j}=\frac{c P n_{j}^{2} e^{2 i \theta}}{2}-\frac{c P}{4} h_{j} d_{j}, \\
b_{j}=\frac{P}{4}\left\{\frac{h_{j} d_{j}-2 n_{j}^{2} \cos 2 \theta}{1-h_{j} d_{j}}+i \frac{2 n_{j}^{2} \sin 2 \theta}{1+h_{j} d_{j}}\right\} .
\end{array}\right\}
$$

Then Eq.(4.26) becomes

$$
\phi(\zeta)=\frac{c P}{2} \zeta^{-1} e^{2 i \theta}+\sum_{i=1}^{2} \frac{L_{j}}{n_{j}-\zeta},
$$

where,

Also,

$$
L_{j}=\frac{c P}{4} h_{j}\left[\frac{1-2 n_{j}^{2} \cos 2 \theta}{1-h_{j} d_{j}}+i \frac{2 n_{j}^{2} \sin 2 \theta}{1+h_{j} d_{j}}\right] .
$$

$$
B(\zeta)=0 \quad, \quad B=0
$$

then,

$$
\psi(\zeta)=-\frac{c P}{4} \zeta^{-1}-\frac{\omega\left(\zeta^{-1}\right)}{\omega^{\prime}(\zeta)} \varphi_{*}(\zeta)+\sum_{j=1}^{2} \frac{h_{j} \zeta}{1-n_{j} \zeta} \varphi_{*}\left(n_{j}^{-1}\right)
$$

where,

$$
\phi_{*}(\zeta)=\phi^{\prime}(\zeta)+\frac{c P}{4} .
$$

This application discusses the first fundamental boundary value problem of an in fin ite plate stretched at infin ity by the application of a uniform tensile stress of intensity heat in the negative direction of $y$-axis. This plate is weakened by a curvilinear hole $C$ which is free fromstress.
For $n_{1}=0.3, \quad n_{2}=0.25, \quad m_{1}=1+0.02 i, \quad m_{2}=-0.01-i$, $p=0.25$, the stress components $\sigma_{x x}, \sigma_{y y}$ and $\sigma_{x y}$ are obtained in large forms calculated by computer and illustrated in the following two cases :

(i) When the study is in the normal plate, we have the following shapes for the stress components, see Fig.(12) and Fig.(13).

(ii) In the thermoelasticity plate, we have the following shapes for the stress components by using the substitutions $G=\frac{1}{2}, q=0.1, \quad r_{0}=0.75, \alpha=0.7, v=1$, see Fig.(14) and Fig.(15).

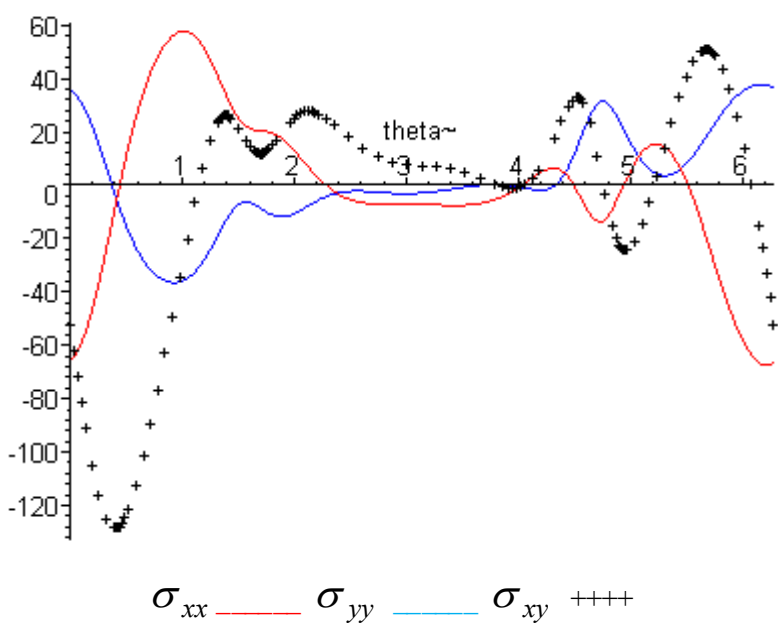

Figure (12)

$\sigma_{x x}$ has positive values at $0.127 \pi \leq \theta \leq 0.7 \pi$, $1.27 \pi \leq \theta \leq 1.4 \pi$ and $1.6 \pi \leq \theta \leq 1.75 \pi$. $\sigma_{y y}$ has positive values at $0 \leq \theta \leq 0.127 \pi$ and $1.37 \pi \leq \theta \leq 2 \pi . \sigma_{x y}$ has positive values at $\frac{\pi}{3} \leq \theta \leq 1.5 \pi$ and $\quad 1.656 \pi \leq \theta \leq 1.94 \pi$
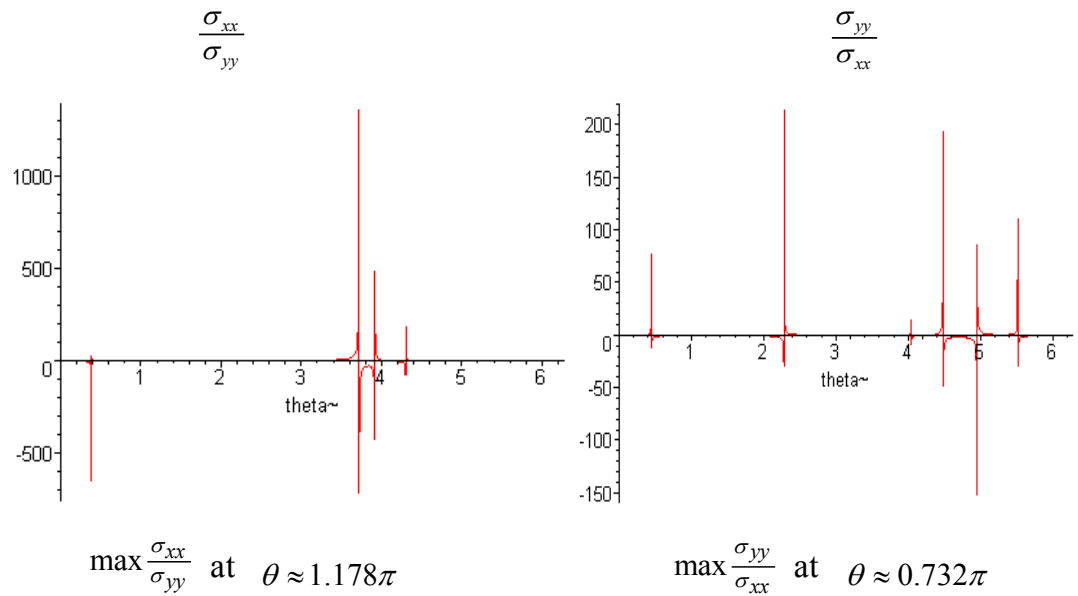

Figure (13) 


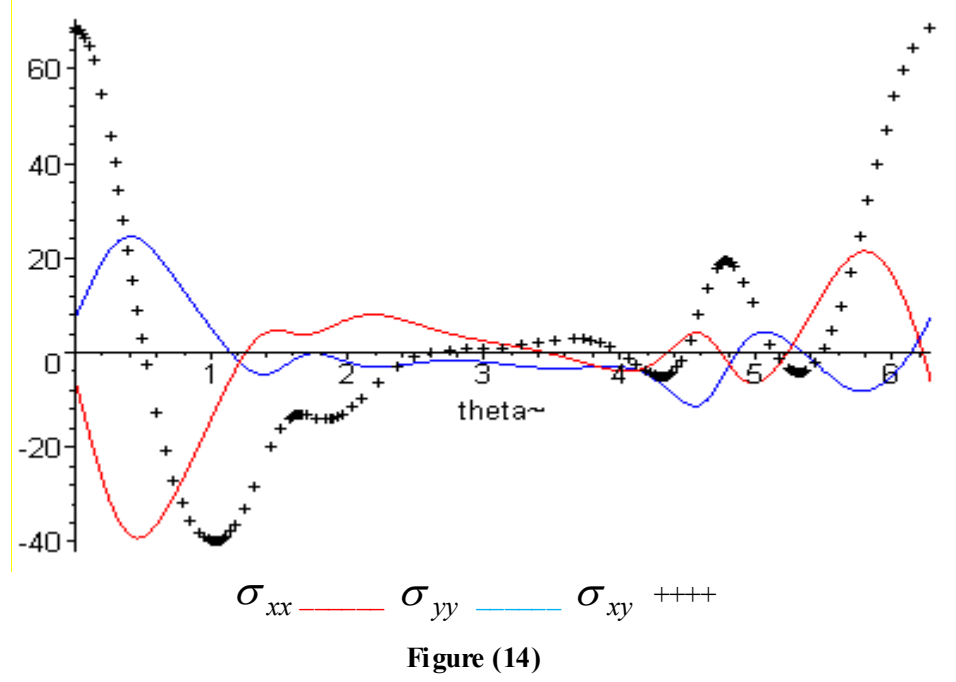

$\sigma_{x x}$ has positive values at $0.38 \pi \leq \theta \leq 1.1 \pi, 1.4 \pi \leq \theta \leq 1.53 \pi$ and $1.656 \pi \leq \theta \leq 2 \pi$. $\sigma_{y y}$ has positive values at $0 \leq \theta \leq 0.38 \pi$ and $1.53 \pi \leq \theta \leq 1.72 \pi . \sigma_{x y}$ has positive values at $0 \leq \theta \leq 0.16 \pi, 0.828 \pi \leq \theta \leq 1.274 \pi, 1.4 \pi \leq \theta \leq 1.656 \pi$ and $1.75 \pi \leq \theta \leq 2 \pi$.
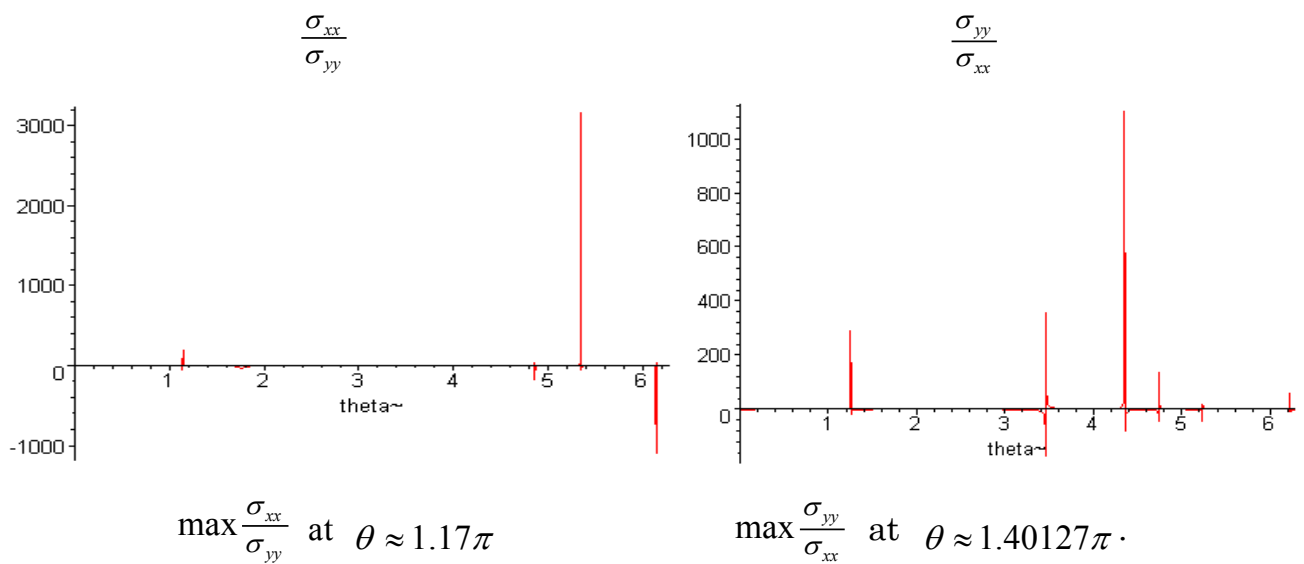

Figure (15)

\subsection{Application 2: Curvilinear Hole Having two Poles and the Edge is Subject to a Uniform Pressure $P$.}

If $K=-1 \quad, S_{x}=S_{v}=\Gamma=\Gamma^{*}=0 \quad, f(t)=P t, \quad \Theta=q y$. Then,

$$
\begin{aligned}
& N(\zeta)=0, \\
& f(\sigma)=P c \frac{\sigma^{3}+m_{1} \sigma+m_{2}}{\left(\sigma-n_{1}\right)\left(\sigma-n_{2}\right)}, \\
& \overline{f(\sigma)}=P c \frac{1+m_{1} \sigma^{2}+m_{2} \sigma^{3}}{\sigma\left(1-n_{1} \sigma\right)\left(1-n_{2} \sigma\right)}, \\
& \overline{A^{\prime}\left(n_{j}\right)}=c P \sum_{j=1}^{2} \frac{n_{j}^{2}+m_{1} n_{j}+m_{2}}{\left(n_{j}-n_{j \pm 1}\right)\left(n_{j} \zeta-1\right)^{2}} \text {. } \\
& E_{j}=-\overline{A^{\prime}\left(n_{j}\right)}, \\
& E_{j}=\bar{E}_{j} \text {, } \\
& b_{j}=-\frac{E_{j}}{c\left(1-h_{j} d_{j}\right)}
\end{aligned}
$$

By using these values Eq.(4.26) becomes

$$
\varphi(\zeta)=c P \sum_{j=1}^{2} \frac{n_{j}^{3}+m_{1} n_{j}+m_{2}}{\left(n_{j}-n_{j \pm 1}\right)\left(n_{j}-\zeta\right)}-\sum_{j=1}^{2} \frac{h_{j} E_{j}}{\left(n_{j}-\zeta\right)\left(1-h_{j} d_{j}\right)} \text {. }
$$

Also,

$$
B(\zeta)=-\frac{c P}{\zeta}
$$

and,

$$
B=\left(n_{1}+n_{2}\right) c P \text {. }
$$

Hence Eq.(4.37) becomes

$$
\begin{aligned}
\psi(\zeta)= & -\frac{\omega\left(\zeta^{-1}\right)}{\omega^{\prime}(\zeta)} \varphi^{\prime}(\zeta)-c P \zeta^{-1}-\left(n_{1}+n_{2}\right) c P \\
& +\sum_{j=1}^{2} \frac{h_{j} \zeta}{1-n_{j} \zeta} \varphi^{\prime}\left(n_{j}^{-1}\right)
\end{aligned}
$$

The previous discussing give the solutions of first fundamental boundary value problem when the edge of hole is subject to a uniform pressure $P$.

For $n_{1}=0.3, \quad n_{2}=0.25, \quad m_{1}=1+0.02 i, \quad m_{2}=-0.01-i$, 
$p=0.25$, the stress components $\sigma_{x x}, \sigma_{y y}$ and $\sigma_{x y}$ are obtained in large forms calculated by computer and illustrated in the following two cases :

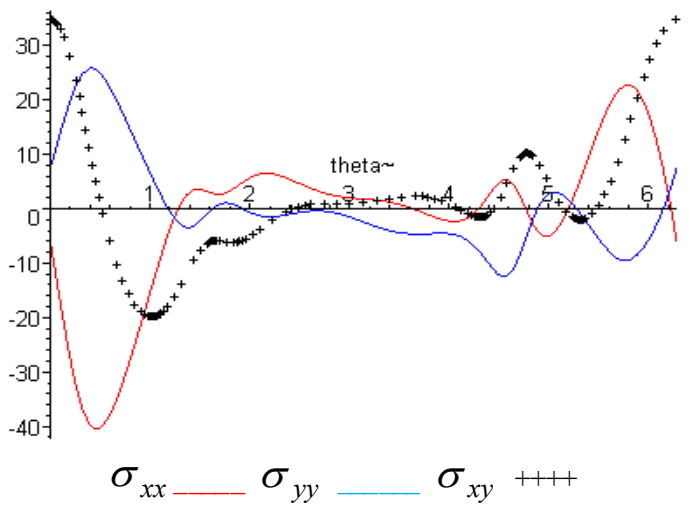

Figure (16)

$$
\frac{\sigma_{x x}}{\sigma_{y y}}
$$

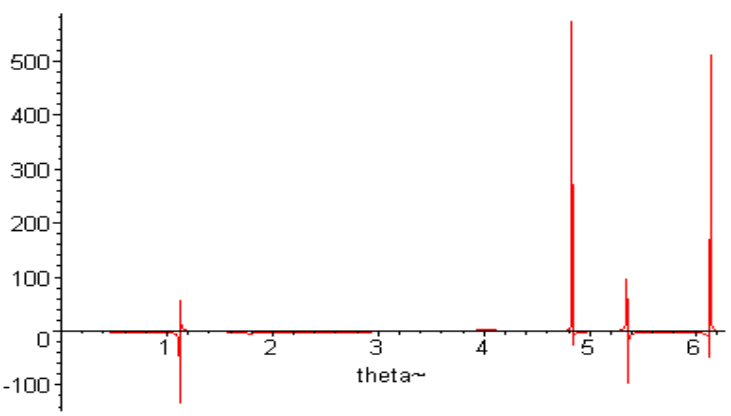

$$
\max \frac{\sigma_{x x}}{\sigma_{y y}} \text { at } \theta \approx 1.52866 \pi \max \frac{\sigma_{y y}}{\sigma_{x x}} \text { at } \theta \approx 1.0987 \pi
$$

Figure (17)

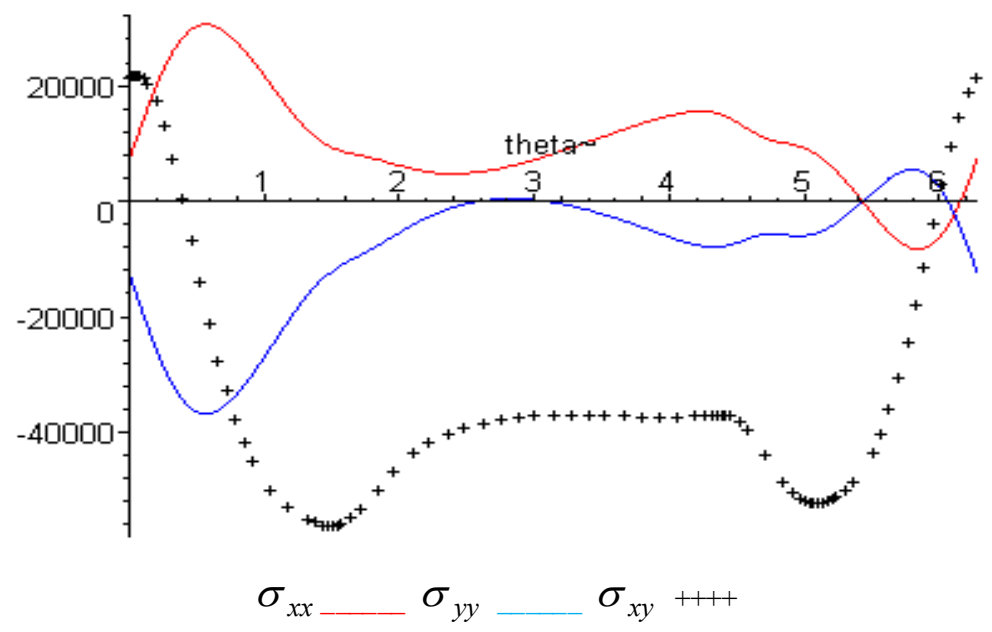

Figure (18)

$\sigma_{x x}$ has positive values at $0 \leq \theta \leq 1.7197 \pi . \sigma_{y y}$ has positive values at $0.828 \pi \leq \theta \leq 0.9554 \pi$ and $1.7197 \pi \leq \theta \leq 1.91 \pi$. $\sigma_{x y}$ has positive values at $0 \leq \theta \leq 0.1274 \pi$ and $\theta \leq 1.91 \pi$. (i) When the study is in the normal plate, we have the following shapes for the stress components, see Fig.(16) and

(ii) In the thermoelasticity plate, we have the following shapes for the stress components by using the substitutions $G=\frac{1}{2}, q=0.1, r_{0}=0.75, \alpha=0.7, v=1$, see Fig.(18) and Fig.(19).

$\sigma_{x x}$ has positive values at $0.38 \pi \leq \theta \leq 1.1 \pi$, $1.4 \pi \leq \theta \leq 1.53 \pi$ and $1.656 \pi \leq \theta \leq 2 \pi . \sigma_{y y}$ has positive values at $0 \leq \theta \leq 0.38 \pi$ and $1.53 \pi \leq \theta \leq 1.72 \pi . \sigma_{x y}$ has positive values at $0 \leq \theta \leq 0.16 \pi, \quad 0.828 \pi \leq \theta \leq 1.274 \pi$, $1.4 \pi \leq \theta \leq 1.656 \pi$ and

$$
1.75 \pi \leq \theta \leq 2 \pi
$$
Fig.(17).
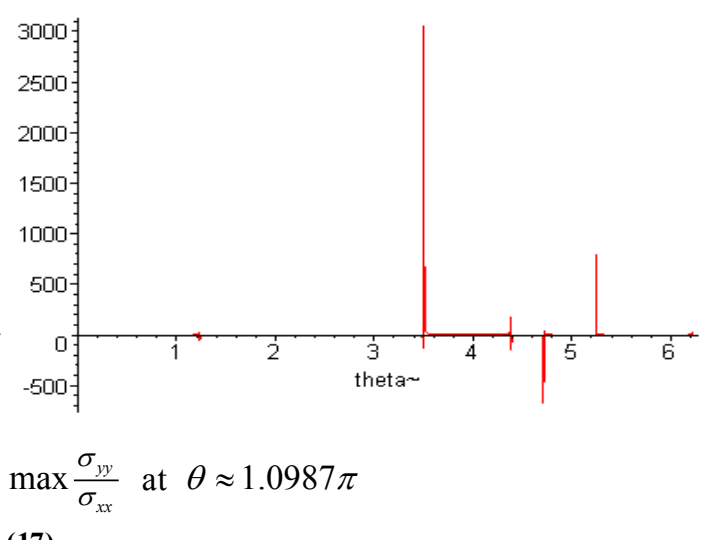

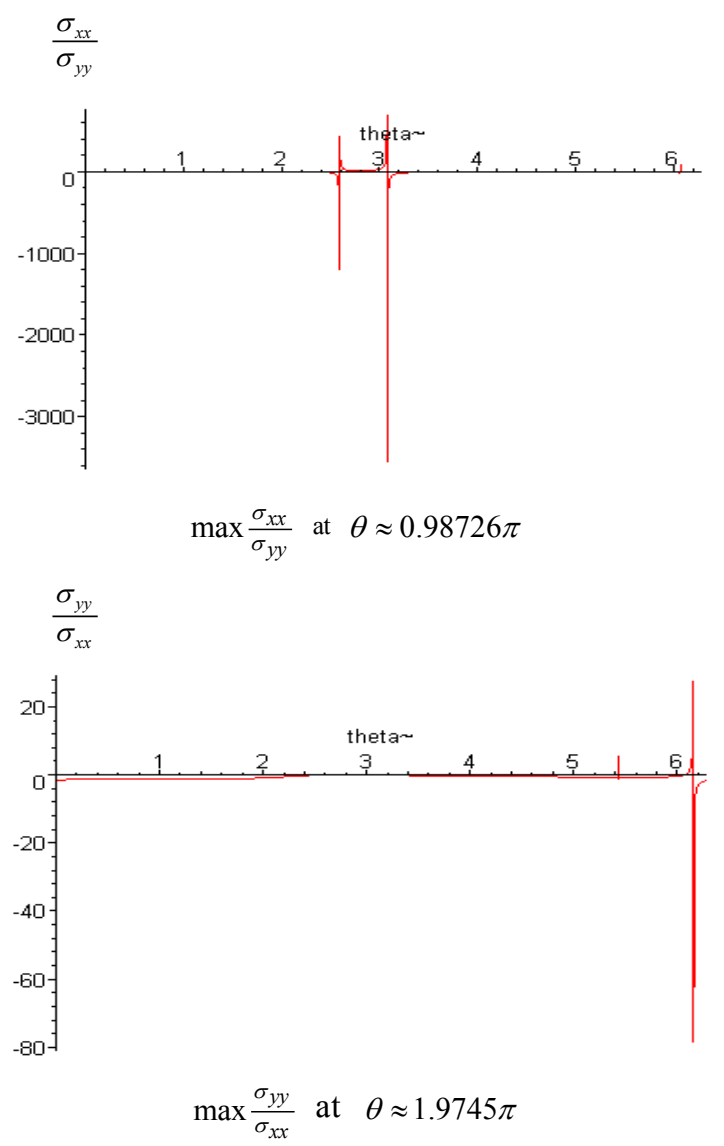

Figure (19)

\subsection{Application 3: The External Force Acts on the Cen- ter of the Curviline ar Hole .}

When, $K=k, \Gamma=\Gamma^{*}=f=0$, then,

$$
\left.\begin{array}{l}
N\left(n_{j}\right)=-\frac{S_{x}-i S_{y}}{2 \pi(1+k)} n_{j}, \\
\overline{N\left(n_{j}\right)}=-\frac{S_{x}+i S_{y}}{2 \pi(1+k)} n_{j} .
\end{array}\right\}
$$

$$
\left.\begin{array}{l}
E_{j}=h_{j} d_{j} n_{j} \frac{S_{x}+i S_{y}}{2 \pi(1+k)}, \\
\bar{E}_{j}=h_{j} d_{j} n_{j} \frac{S_{x}+i S_{y}}{2 \pi(1+k)}
\end{array}\right\}
$$

and,

$b_{j}=\frac{1}{c\left(k^{2}-h_{j}^{2} d_{j}^{2}\right)}\left\{k h_{j} d_{j} n_{j} \frac{S_{x}+i S_{y}}{2 \pi(1+k)}-h_{j}^{2} d_{j}^{2} n_{j} \frac{S_{x}-i S_{y}}{2 \pi(1+k)}\right\}$.

Hence Eq.(4.26) reduce to,

$$
\begin{aligned}
-k \varphi(\zeta)= & \frac{1}{2 \pi(1+k)} \sum_{j=1}^{2} \frac{h_{j} n_{j}}{\left(n_{j}-\zeta\right)} \\
& {\left[\frac{k h_{j} d_{j}\left(S_{x}+i S_{y}\right)}{\left(k^{2}-h_{j}^{2} d_{j}^{2}\right)}-\left(1+\frac{h_{j}^{2} d_{j}^{2}}{\left(k^{2}-h_{j}^{2} d_{j}^{2}\right)}\left(S_{x}-i S_{y}\right)\right)\right] }
\end{aligned}
$$

And the second function given by,

$$
\psi(\zeta)=-\frac{\omega\left(\zeta^{-1}\right)}{\omega^{\prime}(\zeta)} \phi_{*}(\zeta)+\sum_{j=1}^{2} \frac{h_{j} \zeta}{1-n_{j} \zeta} \phi_{*}\left(n_{j}^{-1}\right),
$$

where,

$$
\phi_{*}(\zeta)=\phi^{\prime}(\zeta)-\frac{S_{x}+i S_{y}}{2 \pi(1+k)} \zeta^{-1}
$$

The last application gives the solution of the second fundamental boundary value problem when the heat is flowing in the negative direction of $y$ - axis and the force acts on the centre of the curvilinear hole.

For $n_{1}=0.3, n_{2}=0.25, m_{1}=1+0.02 i, m_{2}=-0.01-i$, $p=0.25$, the stress components $\sigma_{x x}, \sigma_{y y}$ and $\sigma_{x y}$ are obtained in large forms calculated by computer and illustrated in the following two cases:

(i) When the study is in the normal plate, we have the following shapes for the stress components, see Fig.(20) and Fig.(21).

(ii) In the thermoelasticity plate, we have the following shapes for the stress components by using the substitutions $G=\frac{1}{2}, q=0.1, r_{0}=0.75, \alpha=0.7, v=1$, see Fig.(22) and Fig.(23)..

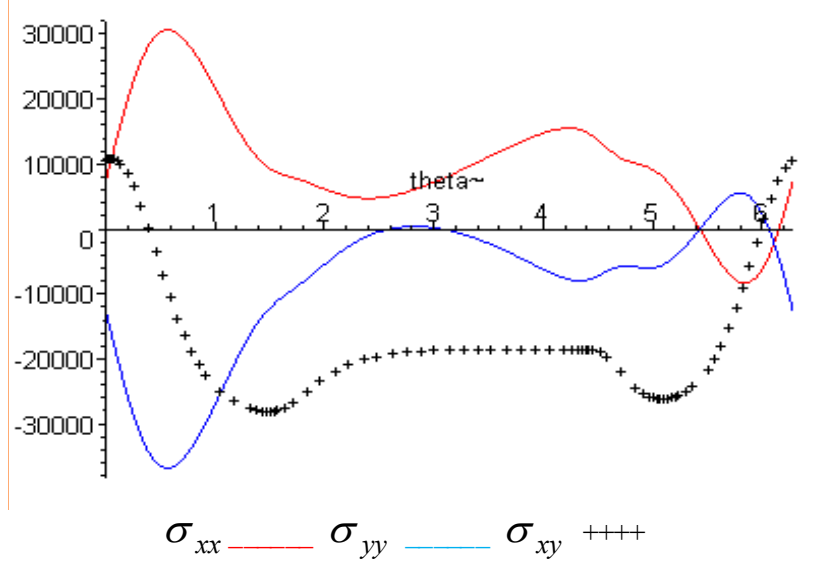

Figure (20) 
$\sigma_{x x}$ has positive values at $0 \leq \theta \leq 1.7197 \pi \cdot \sigma_{y y}$ has positive values at $0.828 \pi \leq \theta \leq 0.9554 \pi$ and $1.7197 \pi \leq \theta \leq 1.91 \pi \cdot \sigma_{x y}$ has positive values at $0 \leq \theta \leq 0.1274 \pi$ and $\theta \leq 1.91 \pi$
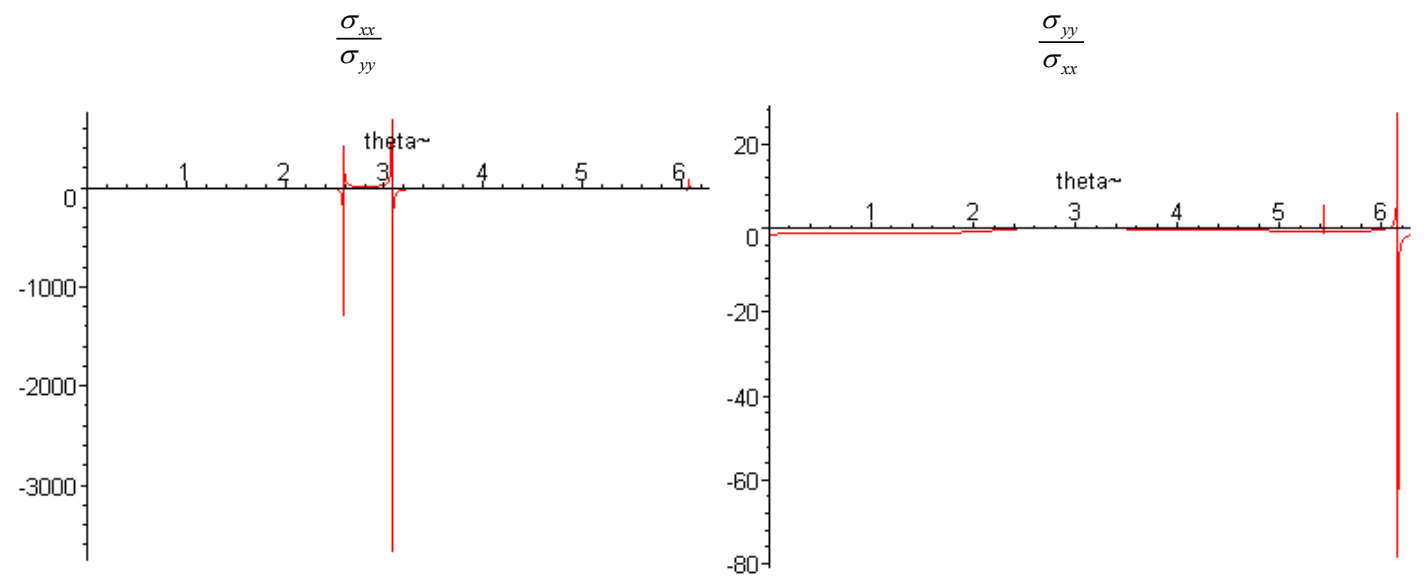

$\max \frac{\sigma_{x x}}{\sigma_{y y}}$ at $\theta \approx 0.9809 \pi \quad \max \frac{\sigma_{y y}}{\sigma_{x x}}$ at $\theta \approx 1.9586 \pi$

Figure (21)

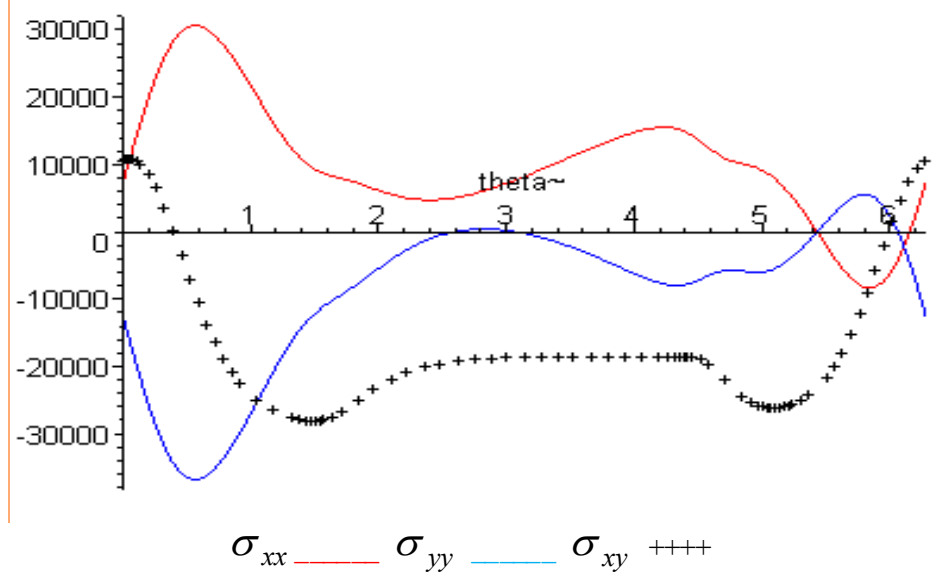

Figure (22)

$\sigma_{x x}$ has positive values at $0 \leq \theta \leq 1.7197 \pi$. $\sigma_{y y}$ has positive values at $0.828 \pi \leq \theta \leq 0.9554 \pi$ and $1.7197 \pi \leq \theta \leq 1.91 \pi \cdot \sigma_{x y}$ has positive values at $0 \leq \theta \leq 0.1274 \pi$ and $\theta \leq 1.91 \pi$.

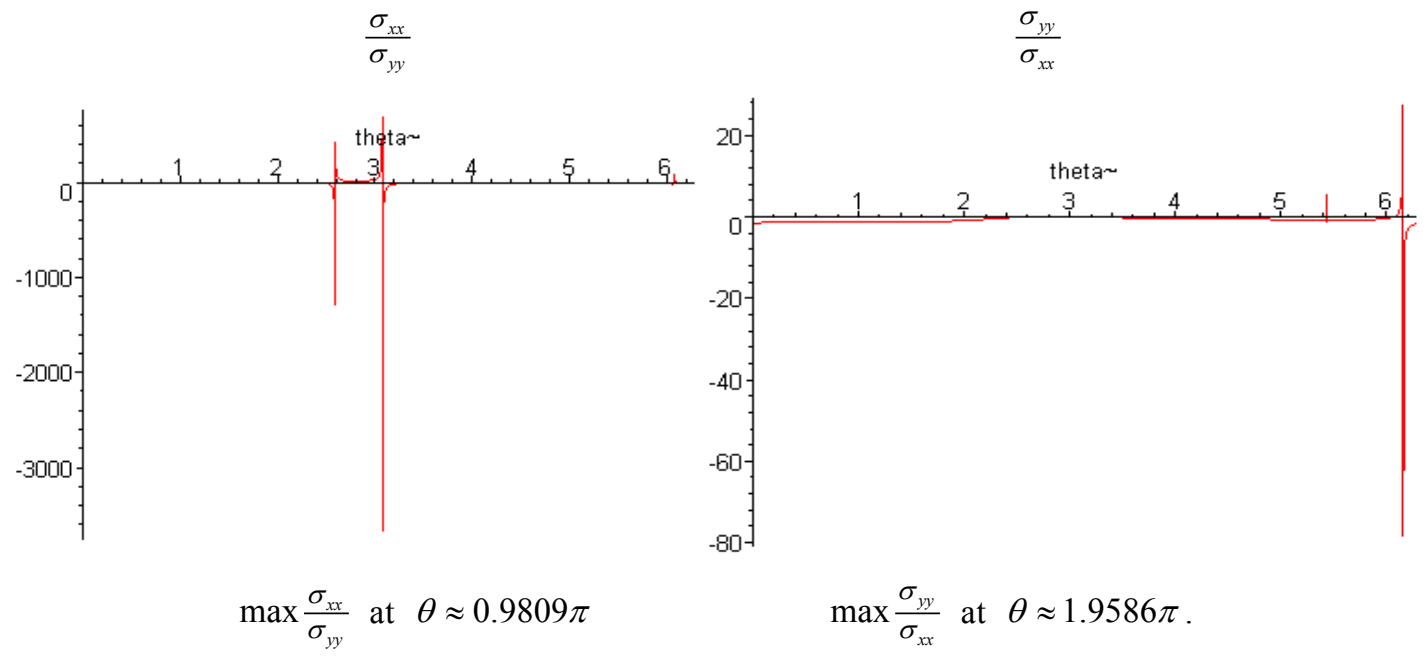

Figure (23) 


\section{Conclusions}

From the previous discussion $\mathrm{s}$ we have the following result

(i) The solution of the boundary value problems for isotropic homogeneous infinite elastic media in $z$-plane reduce to obtain the two complex potential functions, Gaursat functions, in $\zeta$-plane by using conformal mapping.

(ii) The conformal mapping $z=c \omega(\zeta), c>0$, where $\omega^{\prime}(\zeta) \neq 0, \infty$, for $|\zeta|>1$ mapped infinite region to out side a unit circle $\gamma$.

(iii) When we applied the conformal mapping $z=c \omega(\zeta), c>0$ the boundary value problems reduce to the integro-differentail equation with discontinuous kernel.

(iv) Cauchy method is the best method to solving the integro-differentail equation with Cauchy kernel and obtaining the two complex functions $\phi(z)$ and $\psi(z)$ directly.

(v) The components of stress $\sigma_{x x}, \sigma_{y y}$ and $\sigma_{x y}$ is completely determine and plotting after obtaining the two complex functions.

(vi) As a main result of ins erting the effect of uniform flow of heat in the negative of $y$-direction we have, see figures

$$
\max \sigma_{x y}^{N}>\max \sigma_{x y}^{H}
$$

and,

$$
\min \sigma_{x y}^{N}<\min \sigma_{x y}^{H}
$$

where, $\sigma_{x y}^{N}$ represent the shear components of stress at normal state, while $\sigma_{x y}^{H}$ represent the shear components of stress after inserting the effect of heating.

(vii) If we denote to the angle that appears the maximum value of $\frac{\sigma_{x x}}{\sigma_{y y}}$ in normal state by $\theta_{1}$, and the angle that appears the maximum value of $\frac{\sigma_{x x}}{\sigma_{y y}}$ after inserting the effect of heating by $\theta_{2}$, we note

$$
\theta_{1}<\theta_{2}
$$

(viii) If we denote to the angle that appears the maximum value of $\frac{\sigma_{y y}}{\sigma_{x x}}$ in normal state by $\hat{\theta}_{1}$, and the angle that appears the maximum value of $\frac{\sigma_{y y}}{\sigma_{x x}}$ after inserting the effect of heating by $\hat{\theta}_{2}$, we note

$$
\hat{\theta}_{1}<\hat{\theta}_{2}
$$

(ix) All of the previous works in this paper is considered as special cases from this study.

\section{REFERENCES}

[1] F.D.Gakhov, Boundary value problems, Pergamon 1966.

[2] P. G. Ciarlet, M. H. Schultz, and R. S. Varga, Numerical methods of high-order accuracy for nonlinear boundary value problems I. One dimensional problem, Numer. Math., Vol. 9, 1967.

[3] A. Zebib, A Chebyshev method for the solution of boundary value problems, J. Comput. Phys.Vol. 53, 1984.

[4] S.Saito, M.Yamamto, Boundary value problems of quasilinear ordinary differential systems on a finite interval. Math. Jap on. Vol. $34,1989$.

[5] D. Colton, R. Kress, Integral Equation Methods in Scattering Theory, John Wiley, New York, 1983.

[6] M. A. Abdou, First and second fundamental problems for an elastic infinite plate with a curbilinear hole. Alex. Eng. J.Vol. 33, 1994.

[7] M.A. Abdou, Fundamental problems for infinite plate with a curvilinear hole having infinite poles. Appl. Math. Comput.,Vol.93, 2002.

[8] M. A. Abdou and A.K. Khamis, On a problem of an infinite plate with a curvilinear hole having three poles and arbitrary shape. Bull. Call.Math.Soc, Vol.29, 2000.

[9] M.A. Abdou and E.A. Khar-Eldin, An infinite plate weakened by a hole having arbitrary shape. J. Comp. Appl. Math.,Vol. $56,1994$.

[10] N. Noda, R.B. Hetnarski, Y. Tanigawa, Thermal Stress, Taylor and Francis, 2003.

[11] R.B. Hetnarski, J. Ignaczak, Mathematical Theory of Elasticity, Tay lor and Francis, 2004.

[12] H. Parkus, Thermoelasticity, Spring-Verlag, 1976.

[13] G. Ya. Popov, Contact Problems for a Linearly Deformable Foundation, Kiev, Odessa, 1988.

[14] N.I. Muskhelishvili, Some Basic Problems of Mathematical Theory of Elasticity, Holland, 1953.

[15] I.H. El-Sirafy, M.A. Abdou, First and second fundamental problems of infinite plate with a curvilinear hole, J. Math. Phys. Sci.Vol. 18,1984.

[16] H. England, A Complex Variables Method in Elasticity, Dover publications, Inc., New-Youk, 1971.

[17] A.I. Fedotov, Quadrature-difference methods for solving linear and nonlinear singular integro-differential equations, J. Nonlinear Analysis, Vol. 71, 2009.

[18] A. Hanyga, M. Seredyńska, Positivity of Green's functions for a class of partial integro-differential equations including viscoelasticity, J. Wave Motion, Vol. 47, 2010.

[19] V.V. Bavula, The group of automorphisms of the algebra of polynomial integro-differential operators, J. Algebra, Vol.348, 2011.

[20] M.A. AL-Jawary, L.C.Wrobel, Numerical solution of two-dimensional mixed problems with variable coefficients by the boundary-domain integral and integro-differential equation methods, Engineering Analysis with Boundary Elements, Vol.35, 2011. 